\title{
FORMACIÓN EN VIOLENCIA DE GÉNERO: REVISIÓN Y ANÁLISIS EN UNA UNIDAD DOCENTE DE MATRONAS ${ }^{1}$
}

\author{
Mª Jesús Gutiérrez-Martín', Marta Miranda-Gutiérrez², Paula Aller-Franco ${ }^{3}$ \\ Autora correspondencia: $\mathrm{M}^{\mathrm{a}}$ Jesús Gutiérrez-Martín \\ Correo electrónico: gutierrezmartin@ saludcastillayleon.es
}

1. Matrona y tutora coordinadora de la Unidad Docente de Matronas de ValladolidSegovia.

2. EIR matrona de $1^{\circ}$ de la Unidad Docente de Matronas de Valladolid-Segovia.

3. Abogada perteneciente al despacho Lex Negotia de Valladolid y Mediador Judicial.

Recibido: 28/11/2018 Aceptado: 29/01/2019

\footnotetext{
${ }^{1}$ Este trabajo fue presentado como comunicación oral en el III Congreso Internacional «Género, Ética y Cuidado: Visualizando la violencia hacia las mujeres en el embarazo» (Barcelona, 16-18 mayo 2018).
} 


\section{RESUMEN}

\section{Introducción}

El objetivo del presente trabajo es realizar una revisión de la formación teórico-práctica en violencia de género (VG) que se imparte a los Enfermeros Internos Residentes (EIR) de matrona en nuestra unidad docente.

\section{Material y métodos}

Se hizo una revisión de los contenidos sobre VG en la normativa actual y una revisión bibliográfica sobre la formación de profesionales. Se realizó una comparativa con el material y metodología disponibles en nuestra la unidad docente.

\section{Resultados}

Los conocimientos teóricos y adquisición de competencias para la atención a mujeres víctimas de VG queda recogida en la normativa y códigos éticos de las matronas. Se hace necesario prestar especial atención al «currículo oculto». Se definen como metodologías efectivas para mejorar este currículo oculto las actividades autoreflexivas.

\section{Discusión}

La formación en VG en nuestra unidad cumple con los criterios establecidos para la formación de matronas según la normativa vigente. No obstante, los EIR siguen expresando temor y falta de destreza para abordar estas situaciones. Es por ello por lo que hemos planteado dos áreas de mejora.

\section{Conclusiones}

La VG es un problema de salud pública con el que los profesionales muestran inseguridad. Para la formación teórica existen metodologías de fácil implantación, pero para la adquisición de competencias hay que desarrollar el currículo oculto. En nuestra unidad docente se contempla ampliamente la formación en VG, pero parece adecuada la inclusión de informes reflexivos de incidentes críticos en VG.

Palabras clave: violencia contra la mujer; matronas; currículo oculto. 


\section{ABSTRACT}

\section{Introduction}

The purpose of this study is to conduct a review of the theoretical and practical training on violence against women for students of midwifery in our teaching unit.

\section{Material and methods}

A review of the contents related to the topic in the current regulations and a bibliographic review concerning the training of professionals was carried out. Furthermore, a comparison was made between review results and material and methodologies available in our teaching unit.

\section{Results}

Knowledge and competences acquisition on violence against women are reflected in regulations and the ethical codes of midwives. It is necessary work on the «hidden curriculum». Selfreflective activities for improving the hidden curriculum are defined as effective methodologies.

\section{Discussion}

The training on violence against women in our teaching unit fulfills the criteria established by the current regulation of midwives training. However, students of midwifery continue to express anxiety and lack of dexterity to address these situations. We have proposed two improvement areas in this respect.

\section{Conclusions}

The violence against women is a public health issue with which professionals show insecurity. There are methodologies for theoretical training that can be implemented easily. Nevertheless, the hidden curriculum needs to be developed for a successful skill acquisition. The training related to violence against women is widely covered in our Teaching Unit although the inclusion of reports related to critical incidents concerning the violence against women appears as an adequate improvement.

Keywords: violence against women; midwives; hidden curriculum. 


\section{INTRODUCCIÓN}

\section{La VG como problema de salud pública}

La violencia contra la mujer según la ONU (1993) se define como «todo acto de violencia basado en la pertenencia al sexo femenino que tenga o pueda tener como resultado un daño o sufrimiento físico, sexual o psicológico para la mujer, así como las amenazas de tales actos, la coacción o la privación arbitraria de la libertad, tanto si se produce en la vida pública como en la vida privada». La Comisión Europea (2016) defiende que es «una brutal forma de discriminación y una violación de los derechos fundamentales de la víctima. Es causa y consecuencia de desigualdades entre mujeres y hombres».

De manera general, se puede decir que afecta a un $35 \%$ de las mujeres $\mathrm{y}$, específicamente, la violencia contra mujeres por su compañero íntimo al menos una vez su vida, se estima que oscila entre un 10-75\% de mujeres (OMS, 1997). Es un problema que puede comenzar o agravarse durante el embarazo o el puerperio, llegando a alcanzar a un 4\%-8\% de las gestantes en Estados Unidos.

En España es un problema que presenta gran variabilidad geográfica (Ruiz-Pérez, 2010), afectando a un 20\%-48\% de las mujeres que, en mayor medida, sufren violencia psicológica frente a la física, excepto en la Comunidad de Madrid. En cualquier caso, existe una gran variabilidad epidemiológica entre Comunidades Autónomas, posiblemente debido a diferencias socioculturales que condicionan las relaciones de género, diferente desarrollo de las políticas contra la violencia de género, planes de igualdad y género, así como diferencias en la implantación y/o accesibilidad a los recursos sociosanitarios. En el caso concreto de la Comunidad Autónoma, en Castilla y León, la prevalencia de la violencia del compañero íntimo alguna vez en la vida de la mujer oscila entre el 11,5 y el 17\%, siendo menor para la violencia física.

Constituye, por tanto, un problema de salud pública dada su incidencia creciente, su morbilidad y mortalidad (Eberhard-Gran, Schei y Eskild, 2007; Krug et al., 2002). Las consecuencias que la violencia de género (VG) tiene sobre la salud de mujeres y niños son graves (OMS, 2013; Hillis et al., 2016). De los efectos sobre la salud, destaca lo siguiente (Weil, Fletcher y Fenny, 2016):

- La severidad de la violencia se correlaciona con un mayor impacto en la salud física y mental. 
- El impacto de los episodios de violencia repetidos es acumulativo.

- Los efectos de la VG persisten más allá del abuso.

Hay que tener en cuenta, además, que el embarazo es una situación de mayor vulnerabilidad para sufrir VG (Juez, 2008). En la gestación se relacionan con mayor morbimortalidad.

A pesar de la magnitud de este problema, las cifras de denuncias por maltrato representan aproximadamente el 5-10\% de todos los casos que se producen (Cirici-Ameli, Ripoll-Ramos y Querol-Viñas, 2010), aunque según la Secretaría de Políticas de Igualdad, actualmente las denuncias en España han aumentado cerca del 16\%, lo que refleja una mayor sensibilización social sobre el tema (Ruiz-Pérez et al., 2010).

El sector sanitario está integrando su actuación frente a la VG más lentamente que otros sectores (García-Moreno, 2015). Todavía es preciso que este sector fortalezca la actuación contra la VG, incluyendo protocolos y una adecuada y efectiva coordinación entre organismos y redes de apoyo (García-Moreno, 2012). Por ello, entre las estrategias que se plantean para disminuir la incidencia de la VG, se encuentra el fortalecimiento del sector sanitario, por su papel fundamental y su responsabilidad en dar respuesta a este problema (OMS, 2015), así como la formación de sus profesionales para conocer cómo y cuándo actuar (Verdugo Gil et al., 2018) y hacerlo de una manera rutinaria (OMS, 2013). Es importante derribar también barreras como las creencias que tienen muchos profesionales sanitarios al centrar su labor en el tratamiento de enfermedades y sus manifestaciones físicas, considerando tabú su intervención en aspectos privados de las personas a las que atienden (García-Moreno, 2002). En el caso concreto de España, se considera un país que ha legislado respecto a este tema y que se encuentra en proceso de desarrollar protocolos, formar profesionales y establecer indicadores para valorar el progreso e implementación de políticas contra la VG (García-Moreno, 2015).

Dado que las consultas sanitarias de atención primaria y los servicios de urgencias son puntos clave para la detección del problema, la formación de los profesionales sanitarios que trabajan en estos servicios va a ser fundamental (Rodríguez Vega y Fernández Liría, 2002). Estos profesionales deben coordinarse y realizar un trabajo multidisciplinar, donde el personal de enfermería juega un papel fundamental en la detección y atención personalizada a estas mujeres (Griffin y Koss, 2002). 


\section{El papel de la matrona ante la violencia de género}

Recientemente, la OMS ha determinado que las enfermeras y matronas juegan un papel básico en la prestación de servicios de salud seguros, de alta calidad, efectivos y eficaces. Así pues, ha establecido entre sus áreas prioritarias, el desarrollo de la formación y las normativas que permitan y garanticen que sus competencias básicas sean acordes a los principios básicos de Salud 2020, entre los que encontramos principios que abarcan problemas de salud pública tan importantes como la VG. Para este objetivo, la OMS ha establecido como recomendación el desarrollo de programas universitarios y de posgrado que preparen al profesional para que sea capaz de responder a las necesidades de los distintos grupos de pacientes y adaptarse a la evolución de los sistemas sanitarios, lo cual implica un planteamiento centrado en las personas (2016).

Dada la magnitud del problema que supone la VG contra las mujeres en nuestro entorno y siendo fundamental el papel que juegan las matronas en la detección y atención a las víctimas de este problema de salud pública, los objetivos de este trabajo se relacionan a continuación:

1. Objetivo principal: analizar y mejorar la formación en VG que impartimos a los Enfermeros Internos Residentes (EIR) en nuestra Unidad Docente de Matronas de Valladolid-Segovia, con el fin de plantear acciones de mejora si fuera necesario.

2. Objetivos secundarios: para alcanzar el objetivo principal, nos hemos planteado los siguientes objetivos secundarios:

- Realizar una revisión de las competencias y principios éticos que tienen las matronas en VG.

- Realizar una revisión del programa formativo de matronas españolas en VG.

- Revisar las metodologías docentes adecuadas en la formación en VG.

- Comparar los resultados obtenidos en la revisión con la formación en VG que se imparte actualmente en la Unidad Docente de Matronas de Valladolid-Segovia y analizar el grado de satisfacción de los EIR en este aspecto concreto de su formación. 


\section{MATERIAL Y MÉTODOS}

Para el abordaje de los dos primeros objetivos secundarios, se realizó un análisis de los contenidos sobre VG en la normativa española vigente y en el programa oficial de la especialidad que hace referencia a las competencias de las matronas. Del mismo modo, analizamos los códigos deontológicos, tanto nacional como internacional, en referencia al tema de estudio.

Posteriormente, se realizó una revisión bibliográfica desde el 01/01/2018 a 01/03/2018 sobre la formación de profesionales en VG, así como de metodología docente evaluativa que permite mejorar el currículo oculto del EIR y apoyar la evaluación formativa de aspectos tan importantes en el perfil competencial de una matrona como es el manejo de situaciones de VG. Esta búsqueda bibliográfica se realizó a través de la biblioteca sanitaria online de Castilla y León, mediante la cual se accedió a las bases de datos PubMed, UpToDate, ClinicalKey y Dialnet. Las palabras clave utilizadas para la búsqueda fueron «formación violencia género», «midwifery evaluation», «reflective writing» y «critical incidents». Seleccionamos cualquier tipo de estudio o revisión sistemática en relación con el tema a estudio, tanto en lengua española como inglesa. En una primera etapa se seleccionaron 261 resúmenes de estudios, haciendo una segunda selección posterior, tras la que solicitamos el texto completo de 57 trabajos, de los cuales, finalmente, seleccionamos 29 de ellos relacionados con metodologías docentes que trabajan el currículo oculto. El flujograma del proceso de la revisión bibliográfica realizada se refleja en la figura 1. 


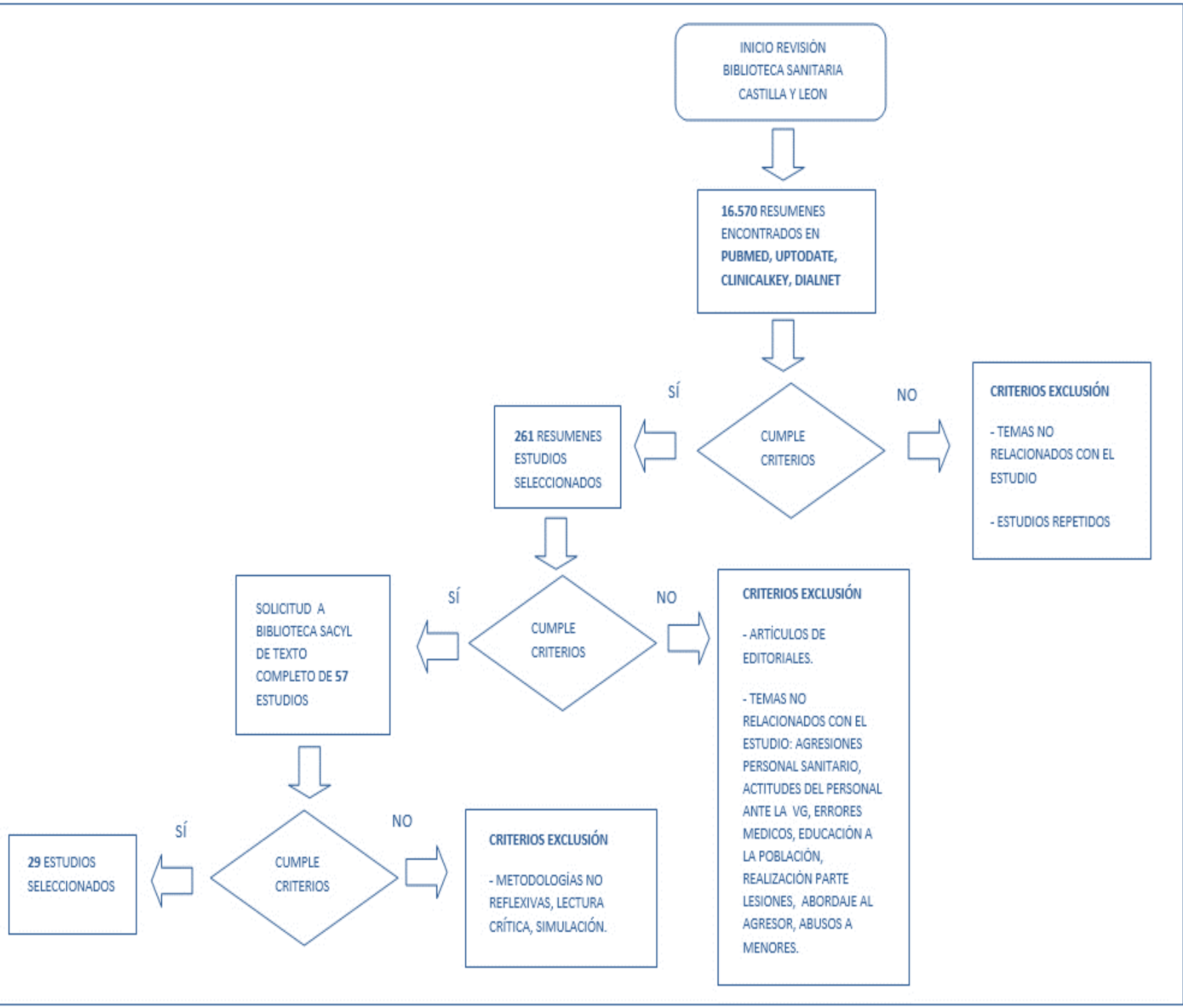

FIGURA 1: Flujograma revisión bibliográfica

Tras la revisión bibliográfica, se realizó una comparativa entre el material disponible dentro de los recursos online con los que cuenta nuestra plataforma de la unidad docente, así como los documentos elaborados por nuestra Subcomisión de Docencia, con las competencias a adquirir y criterios de adquisición de competencias establecidas en la normativa vigente. Igualmente, se valoró la metodología utilizada para la formación de los EIR en VG y se planteó la posibilidad de incluir metodologías de carácter autorreflexivo que mejoren la seguridad de las futuras matronas a la hora de afrontar profesionalmente la atención a mujeres víctimas de VG. 


\section{RESULTADOS}

\section{Competencias que deben adquirir las EIR de matrona}

Existen cuatro normas básicas que reflejan las competencias de las matronas en VG:

1. Directiva 2005/36/CE del Parlamento Europeo y del Consejo, de 7 de septiembre de 2005, relativa al reconocimiento de cualificaciones profesionales (España, 2005).

Esta Directiva fue incorporada al ordenamiento jurídico español en el RD 1837/2008, de 8 de noviembre. Lo establecido en esta norma europea para la matrona, no hace referencia expresa a la atención a mujeres víctimas de VG (Anexo 5.5.1), que únicamente podría considerarse incluida dentro de los apartados definidos para las enseñanzas teóricas y técnicas: «protección jurídica de la madre y el niño (materias básicas) y factores psicológicos y sociales (materias específicas de las actividades de matrona)».

En cualquier caso, esta norma contempla un aspecto importante en su Art. 40: «la lista de materias que figuran en el Anexo $\mathrm{V}$ podrá modificarse con vistas adaptarlas al progreso científico y técnico».

En el caso concreto de nuestro país, las competencias de las matronas en VG sí que se reflejan en el programa oficial de la especialidad, que se comenta a continuación.

2. Orden SAS 1349/2009 de 6 de mayo, por la que se aprueba y publica el programa formativo de la especialidad de Enfermería Obstétrico-Ginecológica (Matrona) (España, 2009). Esta norma hace referencia al papel de la matrona en la atención a la VG en dos apartados:

a) Competencias profesionales (Art. 7):

- Dentro de las competencias en programas de salud materno-infantil, atención a la mujer, salud sexual, salud reproductiva y climaterio, se establece entre los criterios de realización «colaborar con las distintas instituciones para que se realicen políticas de salud con perspectiva de género».

- Dentro de las competencias de atención al embarazo, parto y puerperio, se establecen entre los criterios de realización «detectar situaciones de violencia de género en la mujer gestante, durante el parto y el puerperio, así como atender a las necesidades que presentan las mujeres en situación de violencia de género». 
- Dentro de la competencia de educación para la salud a la mujer, familia y comunidad, establece entre los criterios de realización «contemplar la perspectiva de género en el diseño de programas de salud materno-infantil, salud sexual, reproductiva y climaterio».

- Dentro de las competencias en protección de los derechos de la mujer y su familia, se establecen entre los criterios de realización «identificar y asesorar a la mujer sobre la legislación vigente en materia de violencia de género», así como «detectar situaciones de riesgo relacionadas con infracciones de derechos de la mujer y su entorno; identificar y utilizar los protocolos relacionados con la defensa de los derechos de la mujer y el niño y consultar y derivar a la mujer a otros profesionales en caso necesario».

b) Formación teórica (Anexo I):

- Dentro de la materia enfermería de la mujer y la familia, en el tema 15 , se incluye como materia a impartir la «perspectiva de género, significado social y cultural de la mujer; mujer y educación y violencia contra las mujeres».

- Dentro de la materia educación para la salud de la mujer, se incluye como tema a impartir la «perspectiva de género en los programas de educación para la salud: el constructo de género como determinante de salud; derecho de las mujeres a decidir sobre su salud».

- Dentro de la materia legislación y ética en enfermería obstétrico-ginecológica (matrona), se incluye como materia a impartir los «delitos contra la libertad sexual, agresiones sexuales, violencia de género, abuso sexual, legislación vigente, centros de denuncia y acogida».

3. Código deontológico internacional de matronas (ICM, 2008):

Establece los principios éticos de la práctica de la matronería a nivel internacional, reconociendo a las mujeres como personas con derechos humanos, buscando la justicia para todas las personas y está basado en relaciones de respeto mutuo, confianza y dignidad. Aunque no hace referencia expresa a la VG, es de recalcar que en Art. III.d establece que «las matronas comprenden las consecuencias adversas a la violación de los derechos éticos y humanos sobre la salud de las mujeres y los niños, y trabajarán para eliminar estas violaciones». 
4. Código deontológico de las matronas españolas (AEM, 2011):

Este Código, que se adhiere a lo establecido en el Código Internacional de la Confederación Internacional de Matronas (ICM), sí que hace referencia expresa al papel de la matrona en la atención a las mujeres víctimas de la VG. Declaración previa, apartado c): «Declaramos oposición radical y activa ante cualquier tipo de violencia y particularmente a la violencia de género en cualquiera de sus facetas».

\section{Metodologías docentes adecuadas para formación práctica en VG}

Al igual que muchos otros profesionales sanitarios, las matronas, aunque generalmente están sensibilizadas frente a la VG, no tienen seguridad ni se sienten preparadas para abordar este problema en la práctica clínica (Bradbury-Jones y Broadhurst, 2015; Zambrana Soriano, 2018; Martínez Galiano, 2011). Así pues, sin una adecuada formación previa, estrategias como la realización de entrevistas rutinarias para detectar a mujeres víctimas de VG puede fracasar (Stonard y Whapples, 2016).

Es, por tanto, esencial, plantearnos qué competencias debe desarrollar una futura matrona para poder afrontar de forma efectiva y segura, casos de VG.

1. Competencias a adquirir: Para afrontar estas situaciones se hace necesario prestar especial atención al llamado «currículo oculto», que Epstein (2002) define como «el uso habitual y juicioso de la comunicación, el conocimiento, las habilidades técnicas, emociones, valores y reflexiones de la práctica diaria en beneficio del individuo y la comunidad a la que atiende» (Epstein y Hundert, 2002) y Satterwhite (2000) como «un código no verbalizado de conducta que impregna el entorno y que no es necesariamente congruente con los códigos éticos o de conducta publicados por las organizaciones profesionales y que, a menudo, no se reconoce fácilmente y genera una fuerte dependencia»y que es diferente al «currículo formal» que establecen las normas y programas (Lemp y Seale, 2004).

Entre las competencias que debe adquirir el residente y que pueden mejorar, a su vez, el currículo oculto, encontramos (Potts, 2016):

- Razonamiento clínico: al aplicar los conocimientos teóricos a la práctica clínica, se mejora el pensamiento crítico del personal en formación (Carter, Creedy, Sidebotham, 2015). 
- Habilidades en comunicación y relación con el paciente: permiten que se genere un intercambio de información y colaboración por parte de las mujeres, sus familias y otros profesionales, por lo que también se mejora el trabajo multidisciplinar.

- Profesionalidad: implica mostrar responsabilidad profesional y adherencia a los principios éticos.

2. Metodologías formativas: puesto que nuestro programa formativo orienta al uso de metodologías tanto presenciales como semipresenciales, dando prioridad al aprendizaje activo tutorizado (España, 2009), nos tuvimos que plantear herramientas docentes que permitieran integrar los conocimientos teóricos a la práctica clínica y, por tanto, dirigir el currículo oculto. Para este fin, son útiles metodologías participativas que favorezcan el desarrollo profesional y la reflexión (Ruiz-De Gauna, González-Moro y Morán-Barrios, 2015; Branch, 2001; Walsh, 2010), generando un feedback inmediato y tangible (Kittmer, 2013). Por otra parte, hay que apuntar que se recomienda evitar utilizar únicamente metodologías autorreflexivas, ya que pueden no correlacionarse con metodologías externas y objetivas, por lo que deben utilizarse combinaciones de ambos tipos de métodos (Van Mook y Nka, 2009; Rees and Shepherd, 2005). En cuanto a la reflexión, Boenik (2006) la define como «el examen consciente e intencionado de ideas, sentimientos e implicaciones de la experiencia del aprendizaje; es decir, es una manera de autocrítica constructiva ante incidentes críticos» (2006). La reflexión permite que el personal en formación se conciencie de sus experiencias profesionales para que estas formen parte de su aprendizaje (Westberg y Jason, 2001) y, para un mejor manejo de la misma, debe acompañarse de un feedback constructivo (Shute, 2008).

De entre las metodologías autorreflexivas que pueden mejorar el currículo oculto, destacamos las siguientes:

- Talleres interactivos: habitualmente consisten en una parte teórica inicial complementada con una práctica que puede consistir en un caso clínico, visualización de una película/documental, visualización de una grabación de una consulta real o una dramatización para hacer una reflexión conjunta en pequeños grupos (Branch, 2010; Pohlman, 2013). Es un método que ha demostrado ser 
efectivo para incrementar la confianza de los estudiantes de matrona en la actuación frente a la VG en la gestación (Smith, Wight y Homer, 2018).

- Informes de incidentes críticos mediante reflexiones escritas: Es una metodología ampliamente utilizada como metodología reflexiva y de desarrollo profesional (Walsh, 2010; Charon, 2008; Naber y Markley, 2017). Marcus (1987) considera que incidentes críticos son «las experiencias positivas o negativas que desencadenan pensamientos nuevos o sentimientos que, a menudo, llevan al individuo a pararse a reflexionar y permite examinar sus propias actitudes, valores y creencias» (48). De cara a analizar este tipo de situaciones que surgen habitualmente en la práctica real de las EIR, se trata de una metodología muy útil al poder tratar aspectos que se asocian frecuentemente a casos de VG, como son decisiones éticas, confidencialidad, comunicación con el equipo, seguridad del paciente, nivel de responsabilidad, estrés y emociones (Cohn, 2009). Por otra parte, es también un método que permite al propio tutor ampliar la evaluación formativa de su EIR y mejorar la programación y organización de futuras rotaciones clínicas (Plack, 2010). Existen varios modelos útiles para facilitar la reflexión de una manera estructurada en este tipo de informes (Van Mook y Nka, 2009; Plack, 2010).

- Feedback o devolución constructiva o retroalimentación: Kind, Everett y Ottolini (2009) lo definen como «el acto de dar información a un estudiante o residente, describiendo su desempeño en una situación clínica observada. La disonancia entre el desempeño deseado y el realizado, es un potente motivador que favorece el aprendizaje profundo». Es fundamental utilizarla en este tipo de metodologías, ya que, sin retroalimentación, no se aprende (Schute, 2008). Para llevar a cabo el feedback de manera semiestructurada, se pueden utilizar también diferentes modelos estructurados (De Lima Alves, 2008).

\section{Situación actual en la unidad docente de matronas de Valladolid-Segovia}

En nuestra unidad docente, la formación en VG, se lleva a cabo a través de dos áreas diferenciadas:

1. Adquisición conocimientos teóricos: 
- Conocimientos a través de la plataforma virtual de nuestra unidad docente dentro del campus virtual de Servicio de Salud de Castilla y León (SACYL). En la actualidad se encuentran disponibles más de 50 documentos (artículos científicos, monografías, trabajos científicos, protocolos, normas, etc.).

- Seminarios participativos:

- Seminario sobre perspectiva de género y VG. Se realizan experiencias dramatizadas con reflexión posterior y feedback en grupo.

- Seminario de dudas sobre delitos contra la libertad sexual. Este seminario consiste en la presentación de casos clínicos y reflexión posterior en grupo.

- Seminarios sobre bioética y responsabilidad legal de la matrona. Consistente en una exposición teórica, con una parte final participativa en la que los EIR plantean preguntas y se realiza reflexión en grupo con feedback inmediato.

2. Adquisición de habilidades en la práctica clínica real y/o simulada:

- Evaluación Clínica Objetiva Estructurada (ECOE): esta metodología que consiste en la evaluación de competencias a través de una rueda de estaciones de simulación, permite una valoración objetiva de la adquisición de competencias que realiza un observador mediante un checklist. Tras la realización de la misma, se desarrolla una sesión de debriefing en grupo, como método de retroalimentación constructiva. Posteriormente, los resultados individuales de la prueba se revisan con cada uno de los EIR, momento en el que se realiza también un feedback con carácter individual. Hay que tener también en cuenta, que los informes de la ECOE se trasladan paralelamente al tutor principal del residente, con el fin de que valore si precisa reforzar alguna competencia. Los casos y competencias que se evalúan en cada una de las estaciones los determina el Comité de la ECOE, que recurre habitualmente al diseño de casos de mujeres víctimas de VG en la urgencia ginecológica, urgencia obstétrica y consulta de matrona en atención primaria principalmente.

- Autoaprendizaje Basado en Problemas (ABP): esta metodología docente consiste en la resolución de un caso clínico de la práctica clínica real que abarca diferentes y múltiples campos. Mediante el ABP, se fomenta la búsqueda bibliográfica, la lectura crítica, el razonamiento clínico, la aplicación de conocimientos teóricos a la práctica 
clínica, el trabajo en equipo y las habilidades en comunicación y docencia. Al igual que con la ECOE, la VG es uno de los temas recurrentes en esta metodología.

- Manual de evaluación de competencias: este documento que ha diseñado la unidad docente compila todos los informes y material que se precisan para la evaluación del EIR. Los apartados en los que se puede valorar la adquisición de competencias en VG son los siguientes:

○ Hojas de evaluación de rotaciones/objetivos formativos: en las hojas de evaluación de las rotaciones clínicas por la consulta de matrona en atención primaria, de la consulta de bienestar fetal y en las de urgencias obstétricoginecológicas.

- Entrevistas estructuradas tutor-residente: mediante estas entrevistas estructuradas de carácter autorreflexivo, el tutor refleja si el residente ha adquirido o no las competencias que establece el programa para su año formativo, incluidas las competencias en VG.

- Informe de evaluación formativa: como parte de este informe, se incluyen la valoración de competencias comunes a todas las rotaciones como comunicación, trabajo en equipo, relación con el paciente y bioética. Estas competencias forman parte del currículo oculto del EIR y, por lo tanto y tal como se ha anotado anteriormente, son imprescindibles para que la futura matrona sea capaz de detectar y manejar situaciones de VG.

- Anotación al Plan Individual de Formación del próximo año: mediante esta herramienta, el tutor puede hacer sugerencias de cara a la planificación de la formación específica de sus residentes para el siguiente año. En el caso de detectar alguna deficiencia en la adquisición de competencias requeridas para la adecuada detección y abordaje de situaciones de VG, este informe permite al tutor solicitar rotaciones externas $\mathrm{u}$ otras actividades formativas (trabajos de investigación, cursos, etc.). 


\section{DISCUSIÓN}

\section{Competencias que deben adquirir las EIR de matrona}

Aunque la normativa europea en materia de reconocimiento de cualificaciones profesionales no hace referencia expresa al papel de la matrona en VG, esta materia formaría parte de las competencias en protección jurídica y aspectos psicosociales de la madre.

Sin embargo, la normativa española recoge de forma expresa las competencias en VG para una matrona dentro del programa oficial de la especialidad, tanto a nivel de conocimientos teóricos como a nivel práctico.

\section{Metodologías docentes adecuadas para formación práctica en VG}

De acuerdo con la normativa española para especialistas en Ciencias de la Salud, las unidades docentes debemos adaptar el programa de la especialidad a las características y medios de nuestro Centro Docente a través del itinerario formativo. En el caso de la formación de matronas, se establece un porcentaje de la jornada anual para impartir formación teórico-práctica, además del cronograma de rotaciones que permitan al EIR aplicar esos conocimientos a la práctica real para así adquirir las competencias que requiere una matrona para un ejercicio autónomo de la profesión. En el caso concreto de la VG, es un tema que se debe incluir, por tanto, en los itinerarios formativos.

A pesar de la formación que se imparte a los profesionales, incluidas las matronas, en VG, parece que es una materia que requiere del abordaje de múltiples aspectos, lo cual genera inseguridad y estrés, pudiéndose considerar como un incidente crítico cuando se presenta en la práctica profesional. Es por ello que hay que trabajar el currículo oculto de nuestros residentes, para lo cual se han demostrado efectivas metodologías autorreflexivas que incluyan feedback.

\section{Situación actual en la unidad docente de matronas de Valladolid-Segovia}

El itinerario que hemos desarrollado incluye formación teórico-práctica en materia de VG a través de la plataforma virtual y el desarrollo de seminarios interactivos y seminarios de dudas. El currículo oculto lo trabajamos de una manera general, tanto con el programa teórico como con el cronograma de rotaciones, al recoger todas aquellas competencias 
comunes que abarca, incluidas la profesionalidad, la comunicación, la relación con el paciente y el razonamiento clínico.

La evaluación de las competencias de los EIR en el abordaje de los casos de VG la tenemos contemplada en la práctica real (hojas de evaluación de las rotaciones) y en la práctica simulada (metodología ABP y ECOE).

\section{Procesos de mejora en la formación en VG en la unidad docente de matronas}

Tal y como se ha descrito anteriormente, la detección y asistencia en situaciones de VG queda recogida en la formación y evaluación de nuestros EIR. Esto no es óbice para que ellos sigan expresando temor y falta de destreza a la hora de abordar este tipo de situaciones, lo que se relaciona con lo recogido en la bibliografía consultada (Verdugo Gil, et al.; OMS, 2013).

Desde la Subcomisión de Docencia de Especialidades de Enfermería, órgano colegiado que coordina a los EIR en nuestro centro docente, ante el planteamiento de esta inquietud, se han detectado dos puntos débiles en este sentido:

- La formación de los especialistas en Ciencias de la Salud se centra fundamentalmente en el currículo formal, dejando en un segundo plano el currículo oculto. Con el fin de mejorar las competencias profesionales implicadas en esta área, existen herramientas formativas que han demostrado su utilidad, como son las reflexiones escritas sobre incidentes críticos.

- Si bien en nuestra unidad docente contamos con herramientas que trabajan este aspecto (ECOE, ABP, talleres/seminarios participativos), consideramos que no se tratan de manera individual y pormenorizada las situaciones particulares que vive cada EIR durante su práctica clínica. Por ello, entendemos que los informes sobre incidentes críticos son una herramienta fundamental para que el tutor pueda realizar un feedback individual y constructivo y para que el residente reflexione y desarrolle su currículo oculto. Estos informes serían útiles para situaciones de VG, así como otras situaciones que pueda vivir el residente (duelo perinatal, conflictos con el equipo, conflictos con la paciente/familia, agresiones, demandas judiciales, complicaciones asistenciales, errores en la atención, etc.). 
- Para que la inclusión de este tipo de metodologías sea efectiva, se requiere previamente formación de los tutores que van a utilizarlas. Por ello, antes de su implantación en el libro de residente, la unidad docente deberá solicitar el visto bueno de la Subcomisión de Docencia de Especialidades de Enfermería para posteriormente trabajar el documento de informe estructurado de incidente crítico. Antes de su puesta en marcha, se plantearán seminarios y/o sesiones dirigidas a los tutores para formarles en la reflexión y el feedback constructivo que requiere esta metodología formativa.

\section{CONCLUSIONES}

1. La VG es un problema de salud pública en el que los profesionales sanitarios juegan un papel fundamental. De entre estos profesionales, la matrona destaca como agente básico.

2. A pesar de su importancia y de la existencia de políticas y protocolos de actuación, los profesionales sanitarios no se sienten preparados ni seguros para afrontar situaciones de VG.

3. El currículo formal de las matronas incluye los conocimientos teóricos y competencias en la práctica clínica que tienen que adquirir las EIR para afrontar de forma adecuada este problema de salud.

4. Para la adquisición de los conocimientos teóricos, existen metodologías presenciales y e-learning de fácil implantación.

5. En cuanto a la adquisición de competencias requeridas para formar a los residentes de matrona con el fin de que sean capaces de afrontar situaciones de VG, es recomendable desarrollar el currículo oculto. En este sentido, existen metodologías diversas que favorecen la reflexión y el feedback. Es conveniente combinar varias de ellas (Van Mook y Nka, 2009; Rees y Shepherd, 2005).

6. En el caso concreto de nuestra unidad docente, el tema de VG está ampliamente recogido tanto en el aspecto teórico como en el aspecto práctico, tal y como indica la normativa vigente.

7. No obstante, encontramos que los EIR que formamos siguen adoleciendo de la confianza necesaria para afrontar este problema de salud. A través de este trabajo, se 
plantea la inclusión de informes reflexivos de incidentes críticos y una formación paralela a los tutores antes de su inclusión en el libro del residente, ya que es indispensable su actualización en este sentido para que la metodología sea efectiva (Walsh, 2010; Mabbet et al., 2011).

\section{BIBLIOGRAFÍA}

Asociación EsPaÑOLA DE MATRONAS. Código Deontológico de las Matronas Españolas. AEM, 2011.

BOENINK, Annette Dorothea. Teaching and learning reflection on medical professionalism [tesis doctoral]. Amsterdam: Universidad de Amsterdam, 2006.

BrADBURY-JonES, Caroline; BROADHURST, Karen. Are we failing to prepare nursing and midwifery students to deal with domestic abuse? Findings from a qualitative study. En: Journal of advanced nursing. 2015, vol. 71, núm. 9, pp. 2062-2072.

BRANCH, William T., et al. Teaching the human dimensions of care in clinical settings. En: Jama. 2001, vol. 286, núm. 9, pp. 1067-1074.

BRANCH, William T. The road to professionalism: reflective practice and reflective learning. En: Patient Education and Counseling. 2010, vol. 80, núm. 3, pp. 327-332. Carter, Amanda G.; Creedy, Debra K.; Sidebotham, Mary. Evaluation of tools used to measure critical thinking development in nursing and midwifery undergraduate students: a systematic review. En: Nurse education today. 2015, vol. 35, núm. 7, pp. 864-874.

CHARON, Rita. Narrative medicine: Honoring the stories of illness. Oxford: Oxford University Press, 2008.

CIRICI-AMEli, Roser; Querol-VIÑAS, Núria; RIPOLl-RAMOS, Ana. La consulta sanitaria: ¿un espacio privilegiado para la detección y el abordaje de la violencia de género? En: FMC-Formación Médica Continuada en Atención Primaria. 2010, vol. 17, núm. 8, pp. 550-559.

CoHN, Felicia G., et al. Interpreting values conflicts experienced by obstetricsgynecology clerkship students using reflective writing. En: Academic medicine. 2009, vol. 84, núm. 5, pp. 587-596.

Colombini, Manuela; MAYHew, Susannah; WATTS, Charlotte. Health-sector responses to intimate partner violence in low-and middle-income settings: a review of current 
models, challenges and opportunities. En: Bulletin of the World Health Organization. 2008, vol. 86, pp. 635-642.

COMISIÓN EUROPEA. Resolución sobre una campaña europea sobre tolerancia cero ante la violencia contra las mujeres. Estrasburgo: Parlamento Europeo, 1997.

De Lima Alves, A. E. Constructive feedback. A strategy to enhance learning. En: Medicina. 2008, vol. 68, núm. 1, pp. 88-92.

EBERHARD-GrAnd, Malin; SCHEI, Berit; EsKILD, Anne. Somatic symptoms and diseases are more common in women exposed to violence. En: Journal of general internal medicine. 2007, vol. 22, núm. 12, pp. 1668-1673.

EPSTEIN, Ronald M.; HunderT, Edward M. Defining and assessing professional competence. En: Jama, 2002, vol. 287, núm. 2, pp. 226-235.

EsPaÑA. Directiva 2005/36/CE del Parlamento Europeo y del Consejo, de 7 de septiembre de 2005, relativa al reconocimiento de cualificaciones profesionales. Diario Oficial de la Unión Europea. 30 de septiembre de 2005, L 255, pp. 22-142.

ESPAÑA. Orden SAS/1349/2009, de 6 de mayo por la que se aprueba y publica el programa formativo de la especialidad de Enfermería Obstétrico-Ginecológica (Matrona). BOE. Núm. 129, p. 44697-44729.

GArcíA-Moreno, Claudia, et al. The health-systems response to violence against women. En: The Lancet. 2015, vol. 385, núm. 9977, pp. 1567-1579.

GARCIA-Moreno, Claudia. Dilemmas and opportunities for an appropriate health-service response to violence against women. En: The Lancet, 2002, vol. 359, núm. 9316, pp. $1509-1514$.

GRIFFIN, Michael P.; Koss, Mary P. Clinical screening and intervention in cases of partner violence. En: Online Journal of Issues in Nursing. 2002, vol. 7, núm. 1, p. 2.

HILLIS, Susan, et al. Global prevalence of past-year violence against children: a systematic review and minimum estimates. En: Pediatrics. 2016, vol. 137, núm. 3.

INTERNATIONAL CONFEDERATION OF MIDWIVES. International Code of Ethics for midwives, with explanatory notes and glossary. ICM, 2008.

JUEZ, Velasco. Recomendaciones para la detección precoz de la violencia en el embarazo. En: Matronas profesión. 2008, vol. 9, núm. 3, pp. 32-37.

KInD, Terry; Everett, Veronica R.; OTtolini, Mary. Learning to connect: Students' reflections on doctor-patient interactions. En: Patient education and counseling. 2009, vol. 75, núm. 2, pp. 149-154. 
KITTMER, Tiffaney, et al. Exploring the hidden curriculum: a qualitative analysis of clerks' reflections on professionalism in surgical clerkship. En: The American Journal of Surgery. 2013, vol. 205, núm. 4, pp. 426-433.

KRUG, Etienne G., et al. The world report on violence and health. En: The Lancet. 2002, vol. 360, núm. 9339, pp. 1083-1088.

LEMPP, Heidi; SEALE, Clive. The hidden curriculum in undergraduate medical education: qualitative study of medical students' perceptions of teaching. En: Bmj. 2004, vol. 329, núm. 7469, pp. 770-773.

MABBETT, Gaynor M., et al. Supporting and supervising district nurse students through patchwork text writing. En: Nurse education in practice, 2011, vol. 11, núm. 1, pp. 6-13.

MARCUS, Eric C. Developing Critical Thinkers: Challenging Adults to Explore Alternative Ways of Thinking and Acting. San Francisco: Jossey-Bass, 1988.

Martínez Galiano, Juan Miguel. Detección y atención a la violencia de género en el embarazo: Una asignatura pendiente. En: Enfermería Global. 2011, vol. 10, núm. 24.

NABER, Jessica; MARKLEY, Liz. A guide to nursing students' written reflections for students and educators. En: Nurse education in practice. 2017, vol. 25, pp. 1-4.

ORganización DE LAS NACIONES UnIDAS. Declaración sobre la eliminación de la violencia contra la mujer. Resolución de la Asamblea General 48/104 del 20 de diciembre de 1993. ONU, 1994.

ORgAnización Mundial DE LA SALud (OMS). Global and regional estimates of violence against women: prevalence and health effects of intimate partner violence and non-partner sexual violence. OMS, 2013.

ORGANIZACIÓN MUNDIAL DE LA SALUD (OMS). Global strategy on human resources for health: workforce 2030. OMS, 2016.

ORGANIZACIÓN MUNDIAL DE LA SALUD (OMS). Responding to intimate partner violence and sexual violence against women: WHO clinical and policy guidelines. OMS, 2013.

ORgANiZACión Mundial DE LA SALUd (OMS). World report on ageing and health. OMS, 2015.

PlACK, Margaret M., et al. Peer-facilitated virtual action learning: Reflecting on critical incidents during a pediatric clerkship. En: Academic pediatrics. 2010, vol. 10, núm. 2, pp. 146-152. 
PoHLMAN, Shawn. Reading Ella: Using literary patients to enhance nursing students' reflective thinking in the classroom. En: International journal of nursing education scholarship. 2013, vol. 10, núm. 1, pp. 283-291.

POTTS, John R. Assessment of competence: the accreditation council for graduate medical education/residency review committee perspective. En: Surgical Clinics. 2016, vol. 96, núm. 1, pp. 15-24.

REES, Charlotte; SHEPHERD, Maggie. Students' and assessors' attitudes towards students' self-assessment of their personal and professional behaviours. En: Medical education. 2005, vol. 39, núm. 1, pp. 30-39.

Rodríguez VegA, B.; FERNÁNDEZ LiRÍA, A. La consideración psiquiátrica del maltrato a la mujer. En: Archivos de psiquiatría. 2002, vol. 65, pp. 1-3.

Ruiz-De Gauna, Pilar; González-Moro, Valentín; Morán-Barrios, Jesús. Diez claves pedagógicas para promover buenas prácticas en la formación médica basada en competencias en el grado y en la especialización. En: Educación Médica. 2015, vol. 16, núm. 1, pp. 34-42.

RUIZ-PÉREZ, Isabel, et al. Variabilidad geográfica de la violencia contra las mujeres en España. En: Gaceta Sanitaria, 2010, vol. 24, núm. 2, pp. 128-135.

SAtTerwhite, Robert C.; SAtTerwhite, William M.; EnArson, Cam. An ethical paradox: the effect of unethical conduct on medical students' values. En: Journal of medical ethics. 2000, vol. 26, núm. 6, pp. 462-465.

SHUTE, Valerie J. Focus on formative feedback. En: Review of educational research. 2008, vol. 78, núm. 1, pp. 153-189.

SMITH, Rachel; WIGHT, Raechel; HoMER, Caroline SE. «Asking the hard questions»: Improving midwifery students' confidence with domestic violence screening in pregnancy. En: Nurse education in practice. 2018, vol. 28, pp. 27-33.

StONARD, Gill; WhapPles, Emma. Domestic violence in pregnancy: midwives and routine questioning. En: Pract Midwife. 2016, vol. 19, núm. 1, pp. 26-9.

VAN MoOK, W. N., et al. Approaches to professional behaviour assessment: tools in the professionalism toolbox. En: European journal of internal medicine. 2009, vol. 20, núm. 8, pp. 153-157.

VERDUGO GIL, Elisa, et al. La violencia de género como competencia de enfermería. La importancia de una formación específica. Valladolid: Universidad de Valladolid, Repositorio Documental, 2018. 
WALSH, D. The nurse mentors' handbook. Supporting students in clinical practice. Open University Press, 2010.

WeIL, Amy; FLETCHER, S. W.; FENNY, H. L. Intimate partner violence: Intervention and patient management. En: UpToDate, 2016, vol. 1.

WeStBERG, Jane; JASON, Hilliard. Fostering reflection and providing feedback: Helping others learn from experience. New York: Springer Publishing Company, 2001.

ZAMBrana SoRIANO, Javier. Mejora de la capacitación en violencia de género del personal de enfermería de atención primaria: proyecto de investigación. 2018. 